\title{
Guest Editor Introduction PPoPP 2016, Special Issue 2 of 2
}

\author{
GREY BALLARD, Wake Forest University \\ MARY HALL, University of Utah \\ TIM HARRIS, Oracle Labs \\ BRANDON LUCIA, Carnegie Mellon University
}

\section{ACM Reference format:}

Grey Ballard, Mary Hall, Tim Harris, and Brandon Lucia. 2017. Guest Editor Introduction. ACM Trans. Parallel Comput. 4, 1, Article 1 (August 2017), 2 pages.

https://doi.org/10.1145/3108141

This pair of special issues contains revised versions of eight articles that were originally presented at the 21st ACM SIGPLAN Symposium on Principles and Practice of Parallel Programming (PPoPP 2016), held March 12-16, 2016, in Barcelona, Spain. With eight papers selected this year, we have two special issues to maintain a reasonable size. The four articles in this issue concern sharedmemory algorithms and synchronization:

In "DomLock: A New Multi-Granularity Locking Technique for Hierarchies" the authors present a new locking mechanism for hierarchical data structures. In this setting, locks can be taken on different nodes, with a lock on a parent node conflicting with locks on its children. DomLock introduces a technique for locking multiple nodes at once by acquiring a lock on a selected "dominator" node which grants access to them all.

The article "Lease/Release: Architectural Support for Scaling Contended Data Structures" examines the hardware/software interface used for writing shared-memory data structures. It proposes a "lease" abstraction, allowing a thread to obtain exclusive access for a short bounded period of time-this can reduce contention by giving threads time to use a cache line productively before the line is transferred to another thread.

In "Hybridizing and Relaxing Dependence Tracking for Efficient Parallel Runtime Support" the authors consider techniques for tracking meta-data used in settings such as record-replay tools, race detectors, and transactional memory. The article contrasts several alternatives, both in terms of their interaction with workloads and in terms of their performance in experimental evaluation.

Finally, the article "ESTIMA: Extrapolating ScalabiliTy of In-Memory Applications" describes a tool for predicting the scalability of an in-memory workload based on profiling information

Authors' addresses: G. Ballard, Department of Computer Science, Wake Forest University, PO Box 7311, Winston-Salem, NC 27109; email: ballard@wfu.edu; M. Hall, School of Computing, MEB 3466, University of Utah, Salt Lake City, UT 84112; email: mhall@cs.utah.edu; T. Harris, Oracle, Newton House, Cambridge, UK, CB1 3RQ; email: timothy.l.harris@oracle.com; B. Lucia, Robert Mehrabian Collaborative Innovation Center (CIC), Carnegie Mellon University, 4720 Forbes Avenue, Pittsburgh, PA 15213; email: blucia@andrew.cmu.edu.

Permission to make digital or hard copies of part or all of this work for personal or classroom use is granted without fee provided that copies are not made or distributed for profit or commercial advantage and that copies bear this notice and the full citation on the first page. Copyrights for third-party components of this work must be honored. For all other uses, contact the Owner/Author.

2017 Copyright is held by the owner/author(s).

2329-4949/2017/08-ART1

https://doi.org/10.1145/3108141 
collected at low thread counts. The key idea is to measure the reasons for stalled cycles: the authors observe that extrapolating from these detailed measurements is significantly more effective than simply extrapolating execution times.

We would like to thank the numerous people who made these special issues possible, especially the authors for their extra work in extending their papers and carefully taking into account the reviewers' feedback; the reviewers for their thoughtful comments; Editor-in-Chief Phil Gibbons for suggesting this special issue; the ACM publication staff for making the whole process run smoothly; and the entire PPoPP organizing and steering committees. 